Article

\title{
Bernoulli Polynomials and Their Some New Congruence Properties
}

\section{Ran Duan and Shimeng Shen *}

School of Mathematics, Northwest University, Xi'an 710127, China; duan.ran.stumail@stumail.nwu.edu.cn

* Correspondence: millieshen28@163.com

Received: 18 February 2019; Accepted: 8 March 2019; Published: 11 March 2019

check for updates

\begin{abstract}
The aim of this article is to use the fundamental modus and the properties of the Euler polynomials and Bernoulli polynomials to prove some new congruences related to Bernoulli polynomials. One of them is that for any integer $h$ or any non-negative integer $n$, we obtain the congruence $B_{2 n+1}(2 h) \equiv 0 \bmod (2 n+1)$, where $B_{n}(x)$ are Bernoulli polynomials.
\end{abstract}

Keywords: Euler polynomials; Bernoulli polynomials; elementary method; identity; congruence

MSC: $11 \mathrm{~B} 68 ; 11 \mathrm{~A} 07$

\section{Introduction}

As usual, for the real number $x$, if $m \geq 0$ denotes any integer, the famous Bernoulli polynomials $B_{m}(x)$ (see [1-4]) and Euler polynomials $E_{m}(x)$ (see [2-5]) are decided by the coefficients of the series of powers:

$$
\frac{z \cdot e^{z x}}{e^{z}-1}=\sum_{m=0}^{\infty} \frac{B_{m}(x)}{m !} \cdot z^{m}
$$

and:

$$
\frac{2 e^{z x}}{e^{z}+1}=\sum_{m=0}^{\infty} \frac{E_{m}(x)}{m !} \cdot z^{m}
$$

If $x=0$, then $E_{m}=E_{m}(0)$ and $B_{m}=B_{m}(0)$ are known as the $m^{\text {th }}$ Euler numbers and $m^{\text {th }}$ Bernoulli numbers, respectively. For example, some values of $B_{m}$ and $E_{m}$ are $B_{0}=1, B_{1}=-\frac{1}{2}, B_{2}=\frac{1}{6}$, $B_{3}=0, B_{4}=-\frac{1}{30}, B_{5}=0, B_{6}=\frac{1}{42}$ and $E_{0}=1, E_{1}=-\frac{1}{2}, E_{2}=0, E_{3}=\frac{1}{4}, E_{4}=0, E_{5}=-\frac{1}{2}$, $E_{6}=0$, etc. These polynomials and numbers occupy a very important position in number theory and combinatorics; this is not only because Bernoulli and Euler polynomials are well known, but also because they have a wide range of theoretical and applied values. Because of this, many scholars have studied the properties of these polynomials and numbers, and they also have obtained some valuable research conclusions. For instance, Zhang Wenpeng [6] studied a few combinational identities. As a continuation of the conclusion in [6], he showed that if $p$ is a prime, one can obtain the congruence expression:

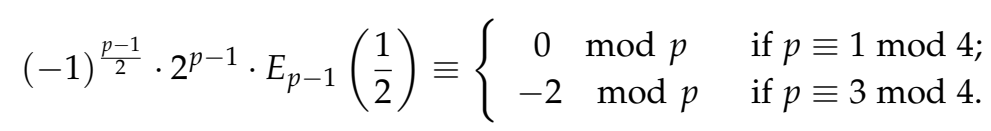

Hou Yiwei and Shen Shimeng [3] proved the identity:

$$
E_{2 n-1}=-\frac{\left(2^{2 n}-1\right)}{n} \cdot B_{2 n}
$$


As some corollaries of [3], Hou Yiwei and Shen Shimeng obtained several interesting congruences. For example, for $p$ in an odd prime, one can obtain the expression:

$$
E_{\frac{p-3}{2}} \equiv 0(\bmod p), \text { if } p \equiv 1 \bmod 8 .
$$

Zhao Jianhong and Chen Zhuoyu [7] obtained the following deduction: if $m$ is a positive integer, $k \geq 2$, one obtains the equation:

$$
\sum_{a_{1}+a_{2}+\cdots+a_{k}=m} \frac{E_{a_{1}}}{\left(a_{1}\right) !} \cdot \frac{E_{a_{2}}}{\left(a_{2}\right) !} \cdots \frac{E_{a_{k}}}{\left(a_{k}\right) !}=\frac{2^{k-1}}{(k-1) !} \cdot \frac{1}{m !} \sum_{i=0}^{k-1} C(k-1, i) E_{m+k-1-i}
$$

for which the summation is taken over all $k$-dimensional nonnegative integer coordinates $\left(a_{1}, a_{2}, \cdots, a_{k}\right)$ such that the equation $a_{1}+a_{2}+\cdots+a_{k}=m$, and the sequence $\{C(k, i)\}$ is decided as follows: for any integers $0 \leq i \leq k, C(k, k)=k !, C(k, 0)=1$,

$$
C(k+1, i+1)=C(k, i+1)+(k+1) C(k, i), \text { for all } 0 \leq i<k,
$$

providing $C(k, i)=0$, if $i>k$, and $k$ is a positive integer.

T.Kim et al. did a good deal of research work and obtained a series of significant results; see [5,8-14]. Specifically, in [5], T. Kim found many valuable results involving Euler numbers and polynomials connected with zeta functions. Other papers in regard to the Bernoulli polynomials and Euler polynomials can be found in [15-19]; we will not go into detail here.

Here, we will make use of the properties of the Euler numbers, Euler polynomials, Bernoulli numbers, and Bernoulli polynomials to verify a special relationship between the Bernoulli polynomials and Euler polynomials. As some of the applications of our conclusions, we also deduce two unusual congruences involving the Bernoulli polynomials.

Theorem 1. For any positive integers $m$ and $h$, the following identity should be obtained, that is:

$$
2 \cdot B_{2 m+1}(2 h)=(2 m+1) \cdot\left(E_{2 m}(2 h)+2 \sum_{i=0}^{2 h-1} E_{2 m}(i)\right) .
$$

Theorem 2. For any positive integers $m$ and $h$, we derive the identity as below:

$$
B_{2 m}(2 h)-B_{2 n}+m\left(E_{2 m-1}(2 h)-E_{2 m-1}\right)=(2 m) \cdot \sum_{i=1}^{2 h} E_{2 m-1}(i) .
$$

From these deductions, the following several corollaries can be inferred:

Corollary 1. Let $m$ be a non-negative integer. Thus, for any integer $h$, we obtain the congruence:

$$
B_{2 m+1}(2 h) \equiv 0 \bmod (2 m+1),
$$

where $\frac{a}{b} \equiv 0 \bmod k$ implies $(a, b)=1$ and $k \mid a$ for any integers $b(b \neq 0)$ and $a$.

Corollary 2. For any positive integer $m$ and integer $h, 2^{2 m-1} \cdot\left(B_{2 m}(2 h)-B_{2 m}\right)$ must be an integer, and:

$$
2^{2 m-1} \cdot\left(B_{2 m}(2 h)-B_{2 m}\right) \equiv 0 \bmod m .
$$

Corollary 3. For any integer $h$, let $p$ be an odd prime; as a result, we have:

$$
B_{p}(2 h) \equiv 0 \bmod p \text { and } B_{2 p}(2 h) \equiv B_{2 p} \bmod p .
$$


Corollary 4. Let $p$ be an odd prime. In this way, there exits an integer $N$ with $N \equiv 1 \bmod p$ such that the polynomial congruence:

$$
N \cdot B_{p}(x) \equiv(x-2)(x-1) x \cdot(x-p+1) \equiv x \cdot\left(x^{p-1}-1\right) \bmod p .
$$

Some notes: It is well known that congruences regarding Bernoulli numbers have interesting applications in number theory; in particular, for studying the class numbers of class-groups of number fields. Therefore, our corollaries will promote the further development of research in this field. Some important results in this field can also be found in [20-23]. Here, we will not list them one by one.

\section{Several Lemmas}

In this part, we will provide three straightforward lemmas. Henceforth, we will handle certain mathematical analysis knowledge and the properties of the Euler polynomials and Bernoulli polynomials, all of which can be discovered from [1-3]. Thus, they will not be repeated here.

Lemma 1. If $m \geq 0$ is an integer, polynomial $2^{m} \cdot E_{m}(x)$ denotes the integral coefficient polynomial of $x$.

Proof. First, from Definition 2 of the Euler polynomials $E_{m}(x)$, we have:

$$
2 e^{x z}=\left(e^{z}+1\right) \cdot \frac{2 e^{x z}}{e^{z}+1}=\left(1+\sum_{m=0}^{\infty} \frac{1}{n !} \cdot z^{m}\right)\left(\sum_{m=0}^{\infty} \frac{E_{m}(x)}{m !} \cdot z^{m}\right) .
$$

On the other hand, we also have:

$$
2 e^{x z}=2 \cdot \sum_{m=0}^{\infty} \frac{x^{m}}{m !} \cdot z^{m}
$$

uniting (3) and (4), then comparing the coefficients of the power series, we obtain that:

$$
2 x^{m}=E_{m}(x)+\sum_{k=0}^{m}\left(\begin{array}{c}
m \\
k
\end{array}\right) E_{k}(x)
$$

or identity:

$$
2 E_{m}(x)=2 x^{m}-\sum_{k=0}^{m-1}\left(\begin{array}{l}
m \\
k
\end{array}\right) E_{k}(x)
$$

Note that $E_{0}(x)=1, E_{1}(x)=x-\frac{1}{2}$, so from (5) and mathematical induction, we may immediately deduce that $2^{m} \cdot E_{m}(x)$ is an integral coefficient polynomial of $x$.

Lemma 2. If $m$ is a positive integer, the following equation can be obtained:

$$
2^{m} \cdot B_{m}(x)=B_{m}(2 x)-\frac{1}{2} \cdot m \cdot E_{m-1}(2 x) .
$$

Proof. From Definitions 1 and 2 of the Euler polynomials and Bernoulli polynomials, we discover the identity as below:

$$
\begin{aligned}
& \frac{2 z e^{2 x z}}{e^{2 z}-1}=\sum_{m=0}^{\infty} \frac{2^{m} \cdot B_{m}(x)}{m !} \cdot z^{m}=\left(\frac{z \cdot e^{2 x z}}{e^{z}-1}-\frac{z \cdot e^{2 x z}}{e^{z}+1}\right) \\
= & \sum_{m=0}^{\infty} \frac{B_{m}(2 x)}{m !} \cdot z^{m}-\frac{1}{2} \sum_{m=0}^{\infty} \frac{E_{m}(2 x)}{m !} \cdot z^{m+1} .
\end{aligned}
$$


Relating the coefficients of the power series in (6), we obtain:

$$
2^{m} \cdot B_{m}(x)=B_{m}(2 x)-\frac{m}{2} \cdot E_{m-1}(2 x) .
$$

This proves Lemma 2.

Lemma 3. If $m$ is a positive integer, then for any positive integer $M$, we will be able to obtain the identities:

$$
2^{m} \cdot\left(B_{m}(M)-B_{m}\right)=m \cdot \sum_{i=0}^{2 M-1} E_{m-1}(i) .
$$

Proof. On the basis of Definition 2 of the Euler polynomials, we obtain:

$$
\sum_{i=0}^{N-1} \frac{2 z e^{i z}}{e^{z}+1}=\sum_{m=0}^{\infty} \frac{1}{n !}\left(\sum_{i=0}^{N-1} E_{m}(i)\right) \cdot z^{m+1} .
$$

In another aspect, we also obtain:

$$
\begin{aligned}
& \sum_{i=0}^{N-1} \frac{2 z e^{i z}}{e^{z}+1}=\frac{2 z\left(e^{N z}-1\right)}{\left(e^{z}+1\right)\left(e^{z}-1\right)}=\frac{2 z e^{N z}-2 z}{e^{2 z}-1} \\
= & \sum_{m=0}^{\infty} \frac{2^{m} \cdot B_{m}\left(\frac{N}{2}\right)}{m !} \cdot z^{m}-\sum_{m=0}^{\infty} \frac{2^{m} \cdot B_{m}}{m !} \cdot z^{m} .
\end{aligned}
$$

Combining (7) and (8), then comparing the coefficients of the power series, we will obtain:

$$
2^{m} \cdot\left(B_{m}\left(\frac{N}{2}\right)-B_{m}\right)=m \cdot \sum_{i=0}^{N-1} E_{m-1}(i)
$$

Now, Lemma 3 follows from (9) with $N=2 M$.

\section{Proofs of the Theorems}

Applying three simple lemmas in Section 2, we can easily finish the proofs of our theorems. Above all, we study Theorem 1. For any positive integer $m$, from Lemma 2, we have:

$$
2^{2 m+1} \cdot B_{2 m+1}(M)=B_{2 m+1}(2 M)-\frac{2 m+1}{2} \cdot E_{2 m}(2 M) .
$$

Note that $B_{2 m+1}=0$. From Lemma 3, we also have:

$$
2^{2 m+1} \cdot B_{2 m+1}(M)=(2 m+1) \cdot \sum_{i=0}^{2 M-1} E_{2 m}(i) .
$$

Combining (10) and (11), we have:

$$
B_{2 m+1}(2 M)=\frac{2 m+1}{2} \cdot E_{2 m}(2 M)+(2 m+1) \cdot \sum_{i=0}^{2 M-1} E_{2 m}(i) .
$$

Afterwards, we prove Theorem 2. According to Lemma 2 with $x=M$ and $x=0$, we have:

$$
2^{2 m} \cdot B_{2 m}(M)=B_{2 m}(2 M)-m \cdot E_{2 m-1}(2 M)
$$

and: 


$$
2^{2 m} \cdot B_{2 m}=B_{2 m}-m \cdot E_{2 m-1} .
$$

Applying Lemma 3, we also have:

$$
2^{2 m} \cdot\left(B_{2 m}(M)-B_{2 m}\right)=(2 m) \cdot \sum_{i=0}^{2 M-1} E_{2 m-1}(i) .
$$

Combining (12), (13), and (14), we have the identity:

$$
B_{2 m}(2 M)-B_{2 m}=m \cdot E_{2 m-1}(2 M)-m \cdot E_{2 m-1}+2 m \cdot \sum_{i=0}^{2 M-1} E_{2 m-1}(i) .
$$

This proves Theorem 2.

From Lemma 1, we know that all $2^{2 m} \cdot E_{2 m}(i)(i=0,1, \cdots, 2 M)$ are integers, and $\left(2^{2 m}, 2 m+1\right)=1$, so on the basis of Theorem 1, we may directly deduce the congruence:

$$
B_{2 m+1}(2 M) \equiv 0 \bmod (2 m+1) .
$$

Since $B_{2 m+1}(x)$ is an odd function (that is, $B_{2 m+1}(-x)=-B_{2 m+1}(x)$ ), and $B_{2 m+1}=0$, so (15) also holds for any integer $M$ and non-negative integer $m$.

This completes the proof of Corollary 1.

Now, we study Corollary 2. On the basis of Lemma 1, we know that $2^{2 m-1} \cdot E_{2 m-1}(i)$ is an integer for all $1 \leq i \leq 2 M$, so from Theorem 1 , we know that $2^{2 m-1} \cdot\left(B_{2 m}(2 M)-B_{2 m}\right)$ must be an integer, and it can be divided by $m$, that is,

$$
2^{2 m-1} \cdot\left(B_{2 m}(2 M)-B_{2 m}\right) \equiv 0 \bmod m .
$$

Note that $B_{2 m}(x)$ is an even function, and if $M=0$, after that, the left-hand side of (16) becomes zero; thus, the congruence (16) is correct for all integers $M$.

This completes the proof of Corollary 2.

Corollary 3 is a special case of Corollary 1 with $2 m+1=p$ and Corollary 2 with $2 m=2 p$.

Now, we prove Corollary 4 . Since $B_{p}(x)$ is a $p^{\text {th }}$ rational coefficient polynomial of $x$ and its first item is $x^{p}$, from Lemma 3 , we know that the congruence equation $B_{p}(2 x) \equiv 0 \bmod p$ has exactly $p$ different solutions $x=0,1,2, \cdots p-1$, so there exits an integer $N$ with $N \equiv 1 \bmod p$ satisfied with $N \cdot B_{p}(x)$, an integral coefficient polynomial of $x$. From [1] (see Theorem 5.23), we have the congruence:

$$
N \cdot B_{p}(x) \equiv x(x-1)(x-2) \cdot(x-p+1) \bmod p .
$$

This completes the proofs of our all results.

\section{Conclusions}

As we all know, the congruences of Bernoulli numbers have important applications in number theory; in particular, for studying the class numbers of class-groups of number fields. The main results of this paper are two theorems involving Bernoulli and Euler polynomials and numbers and four corollaries (or congruences). Two theorems gave some new equations regarding Bernoulli polynomials and Euler polynomials. As some applications of these theorems, we gave four interesting congruences involving Bernoulli polynomials. Especially, Corollaries 1 and 4 are very simple and beautiful. It is clear that Corollary 4 is a good reference for further research on Bernoulli polynomials.

Author Contributions: All authors have equally contributed to this work. All authors read and approved the final manuscript.

Funding: This work is supported by the NSF (11771351) and (11826205) of P. R. China. 
Acknowledgments: The authors would like to thank the Editor and referee for their very helpful and detailed comments, which have significantly improved the presentation of this paper.

Conflicts of Interest: The authors declare that there are no conflicts of interest regarding the publication of this paper.

\section{References}

1. Apostol, T.M. Introduction to Analytic Number Theory; Springer: New York, NY, USA, 1976.

2. Knuth, D.E.; Buckholtz, T.J. Computation of Tangent, Euler, and Bernoulli numbers. Math. Comput. 1967, 21, 663-688. [CrossRef]

3. Hou, Y.W.; Shen, S.M. The Euler numbers and recursive properties of Dirichlet L-functions. Adv. Differ. Equ. 2018, 2018, 397. [CrossRef]

4. Liu, G.D. Identities and congruences involving higher-order Euler-Bernoulli numbers and polynonials. Fibonacci Q. 2001, 39, 279-284.

5. Kim, T. Euler numbers and polynomials associated with zeta functions. Abstr. Appl. Anal. 2008, $2018,581582$. [CrossRef]

6. Zhang, W.P. Some identities involving the Euler and the central factorial numbers. Fibonacci Q. 1998, 36, 154-157.

7. Zhao, J.H.; Chen, Z.Y. Some symmetric identities involving Fubini polynomials and Euler numbers. Symmetry 2018, 10, 303.

8. Kim, D.S.; Kim, T. Some symmetric identities for the higher-order $q$-Euler polynomials related to symmetry group $S_{3}$ arising from $p$-Adic $q$-fermionic integrals on $\mathbb{Z}_{p}$. Filomat 2016, 30, 1717-1721. [CrossRef]

9. Kim, T. Symmetry of power sum polynomials and multivariate fermionic $p$-Adic invariant integral on $\mathbb{Z}_{p}$. Russ. J. Math. Phys. 2009, 16, 93-96. [CrossRef]

10. Kim, T.; Kim, D.S.; Jang, G.W. A note on degenerate Fubini polynomials. Proc. Jiangjeon Math. Soc. 2017, 20, 521-531.

11. Kim, D.S.; Park, K.H. Identities of symmetry for Bernoulli polynomials arising from quotients of Volkenborn integrals invariant under $S_{3}$. Appl. Math. Comput. 2013, 219, 5096-5104. [CrossRef]

12. Kim, T.; Kim, D.S. An identity of symmetry for the degernerate Frobenius-Euler polynomials. Math. Slovaca 2018, 68, 239-243. [CrossRef]

13. Kim, T.; Kim, S.D.; Jang, G.W.; Kwon, J. Symmetric identities for Fubini polynomials. Symmetry 2018, 10, 219. [CrossRef]

14. Kim, D.S.; Rim, S.-H.; Kim, T. Some identities on Bernoulli and Euler polynomials arising from orthogonality of Legendre polynomials. J. Inequal. Appl. 2012, 2012, 227. [CrossRef]

15. Simsek, Y. Identities on the Changhee numbers and Apostol-type Daehee polynomials. Adv. Stud. Contemp. Math. 2017, 27, 199-212.

16. Guy, R.K. Unsolved Problems in Number Theory, 2nd ed.; Springer: New York, NY, USA, 1994.

17. Liu, G.D. The solution of problem for Euler numbers. Acta Math. Sin. 2004, 47, 825-828.

18. Zhang, W.P.; Xu, Z.F. On a conjecture of the Euler numbers. J. Number Theory 2007, 127, 283-291. [CrossRef]

19. Cho, B.; Park, H. Evaluating binomial convolution sums of divisor functions in terms of Euler and Bernoulli polynomials. Int. J. Number Theory 2018, 14, 509-525. [CrossRef]

20. Wagstaff, S.S., Jr. Prime divisors of the Bernoulli and Euler Numbers. Number Theory Millenn. 2002, 3, 357-374.

21. Bayad, A.; Aygunes, A. Hecke operators and generalized Bernoulli-Euler polynomials. J. Algebra Number Theory Adv. Appl. 2010, 3, 111-122.

22. Kim, D.S.; Kim, T. Some $p$-Adic integrals on $\mathbb{Z}_{p}$ Associated with trigonometric functions. Russ. J. Math. Phys. 2018, 25, 300-308. [CrossRef]

23. Powell, B.J. Advanced problem 6325. Am. Math. Mon. 1980, 87, 836. 